CAHIERS DE

NARRATOLOGIE

\section{Cahiers de Narratologie}

Analyse et théorie narratives

36 | 2019

Rhétorique et représentations de la culture mafieuse. Images, rituels, mythes et symboles

\title{
Don Raffaè de De André/Bubola/Pagani : représentation exemplaire du mafieux généreux aimé par le petit peuple
}

Walter Zidarič

\section{OpenEdition}

\section{Journals}

Electronic version

URL: http://journals.openedition.org/narratologie/9788

ISSN: 1765-307X

Publisher

LIRCES

Electronic reference

Walter Zidarič, «Don Raffaè de De André/Bubola/Pagani : représentation exemplaire du mafieux généreux aimé par le petit peuple », Cahiers de Narratologie [Online], 36 | 2019, Online since 20

December 2019, connection on 21 December 2019. URL : http://journals.openedition.org/narratologie/ 9788

This text was automatically generated on 21 December 2019.

Article L.111-1 du Code de la propriété intellectuelle. 


\title{
Don Raffà̀ de De André/Bubola/ Pagani : représentation exemplaire du mafieux généreux aimé par le petit peuple
}

\author{
Walter Zidarič
}

La chanson Don Raffaè est publiée par Fabrizio De André en 1990 dans son album Le Nuvole (les nuages), le douzième de sa carrière. La musique est l'œuvre de Mauro Pagani alors que le texte est écrit à quatre mains par De André lui-même et Massimo Bubola. Aucun des deux n'est Napolitain : pourtant, Don Raffaè est désormais un grand classique de la chanson parthénopéenne ${ }^{1}$. Pagani a expliqué en ces termes la genèse de cet album qui mettait fin à six années de silence après Crêuza de mä (1984) :

Lorsque nous avons commencé à travailler sur le nouveau disque, nous nous sommes rendu compte, en fait, qu'avec le temps qui s'était écoulé notre relation était devenue plus profonde, que nos connaissances s'entrelaçaient de plus en plus et s'influençaient mutuellement. Ainsi, cette fois-ci, tout a véritablement pris forme et identité en bavardant, en inventant, en faisant et en refaisant à nouveau à quatre mains. Surtout, en regardant autour de nous, avec une attention vers le monde totalement différente de celle qui avait inspiré le disque génois [...] Tout ce que nous avions de nouveau entre les mains trouvait son poids et sa place : à partir des riches athéniens d'Aristophane, si semblables à ceux d'aujourd'hui, à l'indolence d'Oblomov, de l'enchantement mélancolique de Tchaikovsky à la sagesse quelque peu misérablement clownesque et atemporelle du geôlier Pasquale Cafiero ${ }^{2}$.

L'une des dernières interprétations réussies de Don Raffaè, cinématographique cette fois-ci, se trouve dans le film Passione de John Turturro, présenté hors concours lors de la $67^{7 m e}$ édition de la Mostra de Venise, le 4 septembre 2010. Le travail de Turturro est une sorte d'hybridation entre la fiction cinématographique, le vidéoclip et le documentaire musical, avec parfois l'insertion d'images d'archives sonores. S'il manque une véritable trame à la diégèse du film, qui ambitionne de représenter la ville de Naples à travers la musique et la chanson, notamment pour le public américain (et plus généralement anglophone), on pourrait cependant définir le résultat final comme une 
sorte de rapsodie, au sens étymologique du terme, car il s'agit d'un recueil fort sélectif, eu égard de l'abondance du répertoire napolitain, de morceaux et de réflexions de différents auteurs et interprètes ordonnés de manière à former un ensemble unitaire. Un véritable patchwork, «assemblé " par les interventions rapides du réalisateur/ narrateur, nouveau rapsode de la culture musicale et de la chanson napolitaines.

Des vingt scènes ou numéros dont le film est constitué, c'est Don Raffaè qui occupe à mon sens une position centrale et donc stratégique, la numéro 9, délibérément établie par le réalisateur. Ce dernier nous présente une version cinématographique de la chanson entre le comique et la farce : le geôlier est interprété par Max Casella, qui force les traits de la caricature en courant dans tous les sens dans les escaliers du Castel dell'Ovo, la prison, pour porter des tasses de café, comme dans un bar; le personnage de Pasquale Cafiero est incarné physiquement et vocalement par Peppe Barra aux côtés du boss mafieux, en cellule, endimanché selon la tradition cinématographique des boss affiliés à Cosa Nostra.

4 Voici le texte intégral de la chanson ${ }^{3}$ et la traduction simplifiée que j'en propose, avec en italiques certains éléments sur lesquels on reviendra par la suite :

(I)

Io mi chiamo Pasquale Cafiero

E son brigadiero del carcere oinè

Io mi chiamo Cafiero Pasquale

Sto a Poggioreale dal cinquantatrê

E al centesimo catenaccio

Alla sera mi sento uno straccio

Per fortuna che al braccio speciale

C'è un uomo geniale che parla co' me.

Tutto il giorno con quattro infamoni

Briganti, papponi, cornuti e lacché

Tutte l'ore co' 'sta fetenzia

Che sputa minaccia e s'à piglia co' me

Ma alla fine m'assetto papale

Mi sbottono e mi leggo 'o giornale

Mi consiglio con Don Raffaè

Mi spiega che penso e bevimm' 'o café.

Ah che bell' 'o café

Pure in carcere 'o sanno fa

Co' 'a recetta ch'à Ciccirinella

Compagno di cella

$\mathrm{Ci}$ ha dato mammà
Je m'appelle Pasquale Cafiero

et suis brigadier de la prison, vois-tu,

Je m'appelle Cafiero Pasquale

suis à (la prison de) Poggioreale depuis 1953

et à la centième serrure

le soir je suis sur les rotules

Quelle chance qu'au quartier spécifique

Il y ait un homme génial qui parle avec moi.

Toute la journée au milieu de gens infâmes

de brigands, de proxénètes, de cocus et de laquais

tout le temps avec cette pègre

qui crache des menaces et s'en prend à moi

mais, enfin, je m'assieds comme un pape

je me déboutonne et lis le journal

je demande des conseils à Don Raffaè

Il m'explique ce qui me passe par la tête et nous

buvons le café.

Ah qu'il est bon le café

même en prison ils sont capables de le faire

grâce à la recette que Ciccirinella

compagnon de cellule

A eu de sa mère. 
Prima pagina venti notizie

Ventun ingiustizie e lo Stato che fa ?

Si costerna, s'indigna, s'impegna

Poi getta la spugna con gran dignità

Mi scervello e mi asciugo la fronte

Per fortuna c'è chi mi risponde

A quell' uomo sceltissimo e immenso

Io chiedo consenso, a don Raffaé.

Un galantuomo che tiene sei figl

Ha chiesto una casa e ci danno consigli

conseils

Mentre 'o assessore che Dio lo perdoni

pardonne,

'ndrento 'a roullotte ci tiene i visoni

voi vi basta una mossa una voce

c'ha 'sto Cristo ci levan 'a croce

con rispetto s'è fatto le tre

volité 'a spremuta o volite 'o café ?

Ah che bell' 'o cafe

Pure in carcere 'o sanno fa

Co' 'a recetta ch'à Ciccirinell

Compagno di cella

Ci ha dato mammà

Ah che bell' 'o cafe

Pure in carcere 'o sanno fa

$\mathrm{CO}^{\prime}$ ' a recetta di Ciccirinella

Compagno di cella

Preciso a mammà.

A la une il y a vingt nouvelles du jour

vingt-er-un injustices et l'Etat que fait-il ?

Il est consterné, il s' indigne, s'engage

puis il jette l'éponge de manière très digne

je me creuse les méninges et essuie mon fron

par chance j'ai quelqu'un qui me répon

à cet homme très raffiné et immense

Je demande son consentement, à do

Rafface

Un honnête homme qui a six enfants

a demandé un logement et on lui donne des

alors que l'adjoint au Maire, que Dieu lui

Il héberge des visons dans sa roulotte

vous n'avez besoin que de faire un geste,

de dire un mot

et à ce pauvre Christ on lui ôtera sa croix

avec tout mon respect il est déjả trois heures

voulez-vous un jus pressé ou bien un café ?

Ah qu'il est bon le cafe

même en prison ils sont capables de le faire

grâce à la recette que Ciccirinella

compagnon de cellule

A eu de sa mère.

Ah qu'il est bon le café

même en prison ils sont capables de le faire

gràce à la recette de Ciccirinella

compagnon de cellule

Qui est Identique à sa mère.

Cả ci sta l'inflazione, la svalutazione Ici on a l'inflation, la dévaluation et c'est toujours

E la borsa ce l'ha chi ce l'h

les mêmes qui tiennent les cordons de la bourse

Io non tengo compendio che chillo stipendio moi, je n'ai que mon seul salaire

E un ambo se sogno 'a papa et un gain au loto si mon père m'apparait en rêve

Aggiungete mia figlia Innocenza a cela ajoutons que ma fille, Innocence,

Vuỏ marito non tiene pazienza veut se marier, sans attendre,

Non chiedo la Grazia pe' me

Vi faccio la barba o la fate da sé ?

n'est pas pour moi que je demande la grâce

Est-ce que je vous rase ou vous vous rasez tout seul?

Voi tenete un cappotto cammello_ Vous avez un manteau couleur chamean

Che al maxi-processo eravata 'o cchiu bello et lors đu maxi-procès vous étiez le plus beau

Un vestito gessato marrone un costume marron à petites rayures

Cosi ci è sembrato alla televisione $\quad$ c'est ce qui nous a paru en vous regardant à la télé

Pe' 'ste nozze vi prego Eccellenza

M' 'i prestasse pe' fare presenz

Io già tengo le scarpe e 'o gillè

pour ce mariage, je vous prie, (votre) Excellence,

pourriez-vous me les prêter pour que je sois

èlégant

moi, $j$ 'ai déjả les chaussures et le gilet

Gradite 'o Campari o volite 'o café ?

Désirez-vous un Campari ou bien un café ?

Ah che bell' 'o café

Ah qu'il est bon le café

Pure in carcere 'o sanno fa

$\mathrm{Co}^{\prime}$ ' a recetta ch'à Ciccirinella

Compagno di cella

même en prison ils sont capables de le faire

grâce à la recette que Ciccirinella

compagnon de cellule

$\mathrm{Ci}$ ha dato mammà.

A eu de sa mère.

Ah che bell' 'o cafe

Ah qu'il est bon le café

Pure in carcere 'o sanno fa

$\mathrm{Co}^{\prime}$ ' a recetta di Ciccirinella

Compagno di cella

même en prison ils sont capables de le faire

grâce à la recette de Ciccirinella

compagnon de cellule

Preciso a mammà

Qui est Identique à sa mère. 
(IV)

Qui non c'è più decoro le carceri d'oro

Ma chi l'ha mai viste chissà

Chiste so' fatiscienti pe' chisto i fetienti

Se tengono l'immunità

Don Raffaè voi politicamente

Io ve lo giuro sarebbe 'nu santo

$\mathrm{Ma}$ 'ca dinto voi state a pagà

prison

E fora chist'atre se stanno a spassà.

A proposito, tengo 'nu frate

Che da quindici anni sta disoccupato

Chill'ha fatto cinquanta concorsi

Novanta domande e duecento ricorsi

Voi che data conforto e lavoro

Eminenza vi bacio v'imploro

Chillo duorme co' mamma e co' me

Che crema d'Arabia ch'è chisto café.
Ici il n'y a plus de dignité, les prisons en or

personne ne les a jamais vues

Celles-ci sont délabrées et c'est pour ça

que les criminels

gardent leur immunité

don Raffaè, vous, politiquement,

je vous le jure, vous seriez un saint

mais c'est vous qui êtes en train de payer en

Alors que les autres sont dehors et profitent.

Tiens, à propos, j'ai un frère

qui est au chômage depuis quinze ans

il a passé cinquante concours

a posé 90 candidatures et fait 200 recours

vous qui donnez du réconfort et du travail

(votre) Eminence je vous embrasse je vous implore

celui-là dort avec maman et avec moi

Ce café est une véritable crẻme d'Arabie.

5 Le texte est donc composé de quatre blocs de strophes, chacun comprenant une séquence double d'une strophe de 8 vers (ottava doppia) à laquelle, dans les $2^{\text {ème }}$ et $3^{\text {ème }}$ blocs, vient s'ajouter un refrain 'double' de 10 vers, tandis que le $1^{\text {er }}$ bloc comprend 21 vers - avec un refrain 'simple' de 5 vers - et que le dernier bloc n'a pas de refrain. Il s'agit bien d'une structure régulière de base, 16 vers - ottava doppia justement - dans laquelle on insère strophe après strophe certaines variantes au niveau du refrain, qui disparaît finalement en clôture de chanson.

Cette régularité se repère également dans la scansion métrique puisque, si on analyse les blocs de 16 vers (64 vers au total) des 8 strophes (pour plus de facilité indiquées par des chiffres romains entre parenthèses), l'on retrouve en fin de compte une nette majorité de décasyllabes - 25 (I: 8; II : 7; III :6; IV:4) - et de vers de 11 pieds (endecasillabi) - 19 (I: 6; II : 7; III : 2; IV : 4), auxquels s'ajoutent 8 vers de 9 pieds (novenari) (I : $2 ; \mathrm{II}: 1 \mathrm{III}: 3 ; \mathrm{IV}: 2$ ), 5 vers de 13 pieds (III $: 2 ; \mathrm{IV}: 3$ ), 4 vers de 12 pieds (dodecasillabi) (II $: 1 ;$ III $: 2 ;$ IV $: 1$ ) et 3 vers de 8 pieds (ottonari) (III $: 1 ;$ IV $: 2$ ). Le refrain a lui aussi une structure régulière selon le schéma $6,9,10,6$, avec à chaque fois 5 vers dans les 3 premières strophes. La régularité de la scansion métrique est associée à la scansion rythmique, tout aussi régulière - typique de la tarentelle - et aussi aux nombreuses rimes, embrassées (concorsi/ricorsi), internes (fatiscienti/fetienti) et croisées (me/papale/giornale/Raffà).

7 Tout en abordant plusieurs thèmes, le texte met en exergue le personnage qui donne le titre à la chanson, c'est-à-dire le boss mafieux, qui jouit, comme nous allons le voir, d'une aura sacralisée.

Dans la première strophe, on assiste à la présentation que le brigadier Pasquale Cafiero fait de lui-même, en racontant la dure routine qui est la sienne au quotidien, dans la prison napolitaine de Poggioreale où, au milieu d'une faune carcérale infâme, s'élève, pour ainsi dire, la silhouette d'un personnage d'exception : Don Raffaè. 
Dans la deuxième strophe, à travers la lecture régulière du journal, le brigadier relate tout d'abord l'état de la situation politique et sociale italienne, sans y comprendre grand-chose : il bénéficie par chance des explications "éclairées» du boss. Il passe ensuite à un exemple concret de mauvaise gestion des biens publics, à travers le cas d'un père de famille qui vit dans le besoin, avec ses six enfants, et qui a demandé un logement à la mairie (il s'agit en réalité d'une roulotte); sa demande a été rejetée car l'adjoint préposé aux logements, un ignoble profiteur, s'est tout simplement attribué la roulotte pour y faire de l'élevage de visons.

Dans la troisième strophe, Cafiero passe aux commentaires sur la situation économique générale et évoque ensuite sa situation à lui : avec son maigre salaire, il doit pourvoir aux noces de sa fille qui veut absolument se marier bien que lui n'ait rien d'élégant à se mettre pour l'occasion et ne puisse se permettre d'acheter quoi que ce soit.

11 Voici donc surgir sa première requête au mafieux : que don Raffaè lui prête le manteau qu'il portait lors $\mathrm{du}$ « maxi-procès » tenu contre la mafia, afin de pouvoir faire bonne figure lors de la cérémonie de mariage.

12 Dans la dernière strophe, le brigadier passe en revue la terrible situation des prisons en Italie, en livrant sans détours sa vision politique des choses (sans doute après avoir pris l'avis de Don Raffaè), sur un ton populiste à peine voilé qui propose une image du monde à l'envers, grâce à l'intervention de l'homme providentiel: ceux qui sont incarcérés devraient être libres, car ils sont en train de payer pour ceux qui sont réellement responsables de la situation et qui, eux, sont libres et continuent de profiter de la vie grâce à l'immunité (parlementaire).

Dans la vision du brigadier, l'organisation criminelle de type mafieux a définitivement remplacé l'État. Il est lui-même désormais passé de l'autre côté, comme le démontre d'ailleurs sa déférence vis-à-vis de Don Raffaè, que l'on pourrait qualifier d'« ascendante ", puisqu'il le définit une première fois comme " homme génial » (I, 8), puis comme "homme très raffiné et immense » (II, 7) et enfin, lorsqu'il s'adresse directement à lui, dans la troisième strophe, comme " (votre) Excellence " (III, 13). Il finit par lui déclarer ouvertement qu'il serait bel et bien « un saint» $(\mathrm{IV}, 6)^{4}$, ce qui mène tout droit au dernier terme choisi pour s'adresser au mafieux: «(votre) éminence » (IV, 14).

C'est la deuxième fois, à la fin seulement, que Cafiero demande à nouveau un service au boss : trouver une situation pour son frère, au chômage depuis quinze ans, qui vit toujours à sa charge et à celle de sa mère.

Don Raffaè est donc une chanson de dénonciation, mais sur un ton finement ironique. Outre la politique, l'économie, les combines mafieuses, la situation des prisons et une certaine culture comportementale italienne (et pas seulement napolitaine), le texte et la musique font également allusion à d'autres éléments qui en enrichissent la polysémie.

16 Songeons en premier lieu à la musique et au personnage traditionnel de Cicerenella (c'est l'orthographe exacte de ce prénom), qui est évoqué dans le refrain avec une variante graphique qui comporte trois « $\mathrm{i}$ » de suite, redoublant ainsi la syllabe initiale, Ciccirinella, un diminutif dialectal napolitain qui signifie en réalité "petit pois chiche ", dérivation étymologique directe du latin ččer. Par ailleurs, Cicerenella est aussi le titre d'une chanson populaire napolitaine remontant au XVIIIe siècle ${ }^{5}$, qui est une tarentelle du point de vue musical, accompagnée comme cela arrive souvent dans la 
musique du folklore napolitain par les castagnettes et les tambourins, et qui s'est répandue au cours du XIXe siècle sous le titre de Tarantella di Posillipo ${ }^{6}$. Soulignons tout de même que Cicerenella est une (jeune) femme, alors que Ciccirinella dans Don Raffaè est un détenu de la prison de Poggioreale ; la variante graphique du nom indique aussi une transformation sexuelle du personnage qui a son importance, comme nous allons le voir.

De plus, on ne peut passer sous silence le rite du café, rite païen et prosaïque qui contient pourtant un caractère quasiment "sacré » : il s'agit d'un rite incontournable dans la culture napolitaine, bien qu'il puisse prendre des sens et des connotations différents selon les contextes, comme par exemple dans la pièce Questi fantasmi d'Eduardo De Filippo (pensons au monologue initial de Pasquale à l'acte II) ${ }^{7}$ ou bien dans la chanson Na tazzulella 'e café de Pino Daniele ${ }^{8}$. Dans le cadre de Don Raffaè, le café est sublimé par Ciccirinella car placé sur le même plan que l'autre " autorité » suprême, c'est-à-dire la mamma, dont le prisonnier reproduit la recette exacte, infaillible. Toutefois, le texte semble faire allusion à une certaine ambiguïté, grâce justement à la position de l'adjectif preciso (précis) dans les blocs de strophes II et III, qui pourrait bien avoir une valeur d'attribut épithète pour le café autant que pour celui qui le prépare. Dans ce cas, cela finirait par superposer Ciccirinella à la figure maternelle, à en faire un ersatz de la mamma, avec tout ce que cela comporterait, dans une cellule de prison. Enfin, le refrain renvoie, tant du point de vue musical que du point de vue textuel, à un autre morceau célèbre de Riccardo Pazzaglia (pour le texte) et Domenico Modugno (pour la musique), interprété par ce dernier en 1958, 'O café, dont le refrain est le suivant : «Ah! Che bello 'o café ! / Sulo a Napule 'o sanno fà » (Ah qu'il est bon ce café ! / Il n'y a qu'à Naples qu'on sait le faire) ${ }^{9}$.

Cependant, étant donné le caractère polysémique du texte, l'élément du café en prison peut faire allusion, à n'en pas douter, à l'affaire Sindona, en raison aussi du scandale soulevé à l'époque. Michele Sindona (1920-1987), banquier véreux de la mafia ${ }^{10}$ avait en effet été condamné à perpétuité le 18 mars 1986 en tant que commanditaire de l'homicide de l'avocat Giorgio Ambrosoli ${ }^{11}$ et il était mort empoisonné deux jours après pour avoir bu un café au cyanure de potassium dans la prison super sécurisée de Voghera, en Lombardie. Il faut donc faire attention au lieu où l'on boit un café, c'est évident, mais aussi aux gens avec qui on le boit, et la chanson Don Raffaè nous propose une image oléographique du « bon » mafieux, cordial, disponible avec les gens humbles qui l'admirent et sur lesquels il veille comme un (saint) père.

Concernant la genèse du personnage de Don Raffaè et sa possible ressemblance avec le chef de la Camorra Raffele Cutolo, dont De André et Bubola évoquent le nom, le journaliste Mario Luzzato-Fegiz, rapporte les mots de l'auteur génois pendant que ce dernier s'apprête à monter sur scène pour la répétition générale de son concert :

Pour la deuxième fois en quelques mois un fait divers nous rappelle l'une de ses chansons. Dans un premier temps c'était la «professionnelle» de l'amour chassée par les femmes d'un village (comme dans Bocca di Rosa), maintenant il est question de la connivence mafieuse du chef adjoint des agents pénitentiaires d'une célèbre prison, exactement comme ce qui est raconté dans la chanson Don Raffaè. «Ben oui, je suis un prophète, comme Jérémie, comme Isaïe - plaisante De André -. Il n'en fallait pas beaucoup pour imaginer qu'un chef de la camorra eût à sa solde quelqu'un de la prison. Avec les salaires misérables que l'État donne aux gardiens de prison et la forte personnalité de certains chefs de la camorra et de la mafia, il ne faut pas s'étonner si des connivences et des liens profonds se créent. La chanson Don Raffaè faisait allusion à Raffaele Cutolo, mais bien évidemment ni Massimo 
Bubola, co-auteur du morceau, ni moi-même disposions de nouvelles de première main sur sa détention. Imaginez ma surprise lorsque j'ai reçu une lettre de Cutolo qui se félicitait avec moi pour la chanson et qui ajoutait: "Je ne comprends pas comment vous avez fait pour comprendre ma personnalité et ma situation en prison sans m'avoir jamais rencontré ». Il ne s'était pas vexé et il avait aimé le vers «Don Raffaè, vous, politiquement, je vous le jure, vous seriez un saint », et aussi celui où le brigadier lui demande de trouver un emploi à quelqu'un de sa famille. Cutolo avait joint à sa lettre un livre contenant ses propres poésies; deux d'entre elles, au moins, vraiment remarquables. Je lui ai répondu pour le remercier. Récemment [...] il m'a écrit à nouveau; mais cette fois-ci je ne lui ai pas répondu. Une correspondance avec Cutolo ne semble pas être la meilleure chose : il suffit de beaucoup moins pour finir en prison ${ }^{12}$.

20 À d'autres occasions, De André est revenu sur la genèse du personnage et sur la langue utilisée dans le texte, comme le rappelle Doriano Fasoli :

J'ai utilité exprès un dialecte napolitain de cuisine [...] La clé m'a été fournie par Gli Alunni del sole de Marotta, où il y a un certain don Vito Cacace qui est l'intellectuel $\mathrm{du}$ quartier et qui tous les soirs réunit tout le monde et leur lit le journal en expliquant ce qui se passe. Puis, à d'autres endroits, il y a aussi l'attaque contre l'État: l'État s'indigne, s'engage. Ce sont les mots de Spadolini [qui fut premier ministre], lorsqu'il se précipita à Palerme à l'occasion d'un des nombreux massacres mafieux : «Je suis consterné, je suis indigné et je m'engage... $»^{13}$.

21 Soulignons juste que De André commet un lapsus, quant au titre du roman de Giuseppe Marotta, car il ne s'agit pas de Gli Alunni del sole (1952) mais de Gli Alunni del tempo (1960) du même auteur, où don Vito Cacace est un veilleur de nuit qui discute tous les soirs avec ses voisins des nouvelles parues dans le journal, qu'il est le seul à acheter.

22 Toutefois, le personnage de Don Raffaè semble bien s'inspirer du protagoniste de la pièce Il sindaco del rione Sanità d'Eduardo De Filippo, écrite et montée pour la première fois en 1960 au Teatro Quirino de Rome, où le boss s'appelle Antonio Barracano et partage avec le personnage de la chanson tout le paternalisme mafieux à l'égard des faibles et de ceux qui sont exploités par les institutions et qui lui demandent justice.

Comme l'a raconté Eduardo lui-même, le protagoniste de sa pièce avait été inspiré de la vie réelle :

Il s'appelait Campolungo. C'était un homme brun costaud. Il maintenait l'ordre dans son quartier. On allait le voir pour lui demander son avis sur la façon dont on devait régler les différends dans le quartier de la Sanità. [...] Ces Campolungo n'étaient pas des mafieux, ils vivaient de leur métier, c'était des fabricants de meubles. [...] Il venait toujours à toutes les premières dans ma loge. "Est-ce que je vous dérange? », demandait-il. Il s'asseyait, les mains toujours sur sa canne. «Voulezvous une tasse de café ?». Il répondait « Volontiers». Puis il partait ${ }^{14}$.

24 Avec Don Raffaè, De André s'insère donc de plein droit dans la tradition de la chanson napolitaine qui présente la figure du boss sous une lumière positive, populiste et aussi pour certains aspects populaires, qui avait commencé sans doute en 1914, avec Guapparia de Libero Bovio et Rodolfo Falvo.

Cette veine se poursuit, cependant, de nos jours, sur un autre versant: celui de la musique neomelodica, véritable phénomène de société dont le nom qui le caractérise a été utilisé pour la première fois en 1997 par Peppe Aiello et qui, comme le rappelle Mimmo Gianneri :

explose littéralement, médiatiquement parlant, vers la fin des années 1980. Cela se produit en même temps que la naissance de nombreuses chaînes de télévision commerciales qui, contre d'importantes compensations financières, diffusent les 
vidéos des chanteurs qui se font eux-mêmes de la publicité en inscrivant leur numéro de téléphone à l'écran pour se faire appeler par le public ${ }^{15}$.

Mais qui sont ces neomelodici? En fait, ils

proviennent de la même classe sociale que leurs fans, les "petites gens des quartiers ", dont ils chantent les valeurs et les sentiments. Etant donné que les histoires s'inspirent des vicissitudes des personnages des Quartiers-État, on ne doit pas s'étonner si la camorra apparaît dans les chansons comme un élément naturel avec lequel il faut se confronter tous les jours. [...] ils n'écrivent pas pour exporter une image de la ville, mais pour décrire la partie de Naples qui se sent différente, voire même à l'opposé, du reste de la société italienne ${ }^{16}$.

Puisqu'il semblerait qu'une belle voix et un excellent réseau d'amis et de relations familiales suffisent pour pénétrer dans le business des cérémonies civiles et religieuses à Naples et dans sa région - mariages, baptêmes, fêtes de quartier, etc. -, ce mélange permet aux interprètes neomelodici d'entrer en contact avec la camorra. Au début, sans doute, pour gagner de l'argent de manière "honnête » - en chantant - pour produire un premier disque, pour se faire un nom. Toutefois, comme cela a été souligné par l'avocate Sabrina Vitiello, « le temps passant, on a sous-estimé le substrat pourri de la chanson neomelodica». En réalité, au lieu de se contenter de caricaturer ce genre populaire, ce phénomène sociétal, ces interprètes, bref, tout ce qui fait partie de cet univers atypique (ce qui a longtemps empêché qu'une sérieuse réflexion critique sur de sérieuses bases socio-culturelles voie le jour), il est essentiel de rappeler, comme le fait Mme Vitiello, que «l'exaltation de la chanson neomelodica équivaut à une exaltation du crime organisé napolitain $»^{17}$.

8 Et cette exaltation du crime continue à plein rythme de nos jours, avec des textes s'inscrivant à plein titre dans la mouvance neomelodica tels que $\mathrm{Nu}$ latitante de Tommy Riccio (1993), O Killer (1996) de Gino Del Miro (1996) ${ }^{18}$ et Il mio amico camorrista de Alberto Selly et Tommaso D'Angelo $(2006)^{19}$, que Roberto Saviano a définis une « inquiétante trilogie ${ }^{20}$. A ceux-là on pourrait ajouter aussi 'O capoclan (2004), dont le texte a été signé par A. Alfieri et $C$. Nocerino. Contrairement à la tradition précédente dans laquelle s'inscrit Don Raffaè, ces chansons neomelodiche ont perdu le côté sentimental ou ironique et mordant, au second degré en quelque sorte, pour rendre un piètre hommage à des vrais criminels et provoquant, malgré tout, un phénomène d'identification décidément inquiétant.

\section{BIBLIOGRAPHY}

Aiello Peppe (1997), Concerto napoletano : la canzone, dagli anni settanta a oggi, Lecce, Argo.

De Filippo, Eduardo (1961), Il sindaco del rione Sanità, Torino, Einaudi.

De Filippo, Eduardo (1985), Teatro (Filumena Marturano, Questi fantasmi), Milano, CDE.

Fasoli, Doriano (2001), Fabrizio De André: passaggi di tempo: da « Carlo Martello » a « Princesa », Roma, Edizioni Associate. 
Giammusso, Maurizio (1993), Vita di Eduardo, Milano, Mondadori.

Luzzato-Fegiz, Mario (1997), « De André: io fui facile profeta, me lo confermò Cutolo », Corriere della Sera, 12 février, p. 11

Marotta, Giuseppe (1952), Gli alunni del sole, Milano, Bompiani.

Marotta, Giuseppe (1960), Gli alunni del tempo, Milano, Bompiani.

Pagani, Mauro (2006), Il sentiero delle parole, in Vasta 2006, pp. 245-252.

Sanna, Silvia (2009), Fabrizio De André. Storie, memorie ed echi letterari, Monte Porzio Catone, Effepi Libri, 2009

Sanzone, Daniele (2014), Camorra sound, Milano, Magenes, 2014.

Vacalebre, Federico - Murolo, Roberto - Ranieri, Massimo (2002), De André e Napoli : storia

d'amore e d'anarchia, Milano, Sperling \& Kupfer.

Vasta, Giorgio, éd. (2006), Deandreide. Storie e personaggi di Fabrizio De André in quattordici racconti di scrittori italiani, Milano, Rizzoli (coll. BUR).

\section{Filmographie}

Passione, un film di John Turturro, DVD Cinecittà Luce, 2011, PRV20442

http://www.rai.it/dl/RaiTV/programmi/media/ContentItem-7c6b14c7-1b90-4fbf-bb2fd441f414620b-cinema.html

\section{Sitographie}

https://web.archive.org/web/20031114174300/http://www.railibro.rai.it/interviste.asp?id=95 http://www.classicaviva.eu/catalog/product_info.php?manufacturers_id=10\&products_id=248 https://derivatisanniti.com/2017/03/18/faber-dietro-i-testi-dentro-la-storia-don-raffae/ https://web.archive.org/web/20151206081641/http://archiviostorico.corriere.it/1999/gennaio/ 12/QUELL_ULTIMA_TELEFONATA_co_0_990112150.shtml

http://www.ilgiornale.it/news/e-boss-camorra-ringrazi-de-andr.html

https://www.angolotesti.it/N/testi_canzoni_nccp_nuova_compagnia_di_canto_popolare_7965/ testo_canzone_cicerenella_290287.html

https://www.portanapoli.com/Ita/Teatro/cicerenella.html

http://testicanzoni.mtv.it/testi-Pino-Daniele_8912/testo-\%27Na-tazzulella-\%27ecaf\%C3\%A9-37160031

https://www.rockol.it/testi/44769848/domenico-modugno-o-cafe https://www.youtube.com/watch?v=Qu1Vc0ttCMc.

https://lyricstranslate.com/it/domenico-modugno-\%E2\%80\%99o-caf\%C3\%A8-lyrics.html, https://www.monde-diplomatique.fr/mav/130/LEMOINE/51560

http://ricerca.repubblica.it/repubblica/archivio/repubblica/1985/03/17/il-processo-ambrosoliattende-sindona.html

https://www.bibliocamorra.altervista.org/index.php?

option=com_content\&view=article\&id=244:guapparia\&catid=48:biblioteca-musicale\&Itemid=92

http://testicanzoni.mtv.it/testi-Gianni-Vezzosi_13870924/testo-\%270-killer-10441512 
https://corrieredelmezzogiorno.corriere.it/napoli/notizie/cronaca/2011/17-gennaio-2011/ amico-camorrista-neomelodica-se-conosci-boss-non-giudichi-181274657827.shtml

https://www.peacelink.it/sociale/a/31498.html

https://www.controcampus.it/2014/02/neomelodici-significato-e-storia-il-fenomenoneomelodici-e-la-camorra/

https://www.rockit.it/articolo/neomelodici-tutto-quello-che-ce-sapere

\section{NOTES}

1. La chanson a été enregistrée avec Roberto Murolo, qui l'a insérée dans son album de duos Ottantavoglia di cantare de 1992. Murolo et De André l'ont en outre chantée ensemble en 1992, durant le traditionnel concert du $1^{\mathrm{er}}$ mai. Toutes les traductions ici présentes sont le fait de l'auteur.

2. Pagani 2006, p. 251.

3. Vasta 2006, pp. 201-204.

4. Pour ma part, je suis convaincu qu'il s'agit ici d'un emprunt à la pièce Filumena Marturano d'Eduardo De Filippo, notamment au dernier acte, lorsque Rosalia, en parlant de l'ancienne prostituée, dit à Domenico : «Na santa, vi pigliate, na santa » (C'est une sainte que vous épousez, une sainte), cf. De Filippo 1985, p. 202.

5. Pour texte intégral de la chanson, voir https://www.angolotesti.it/N/ testi_canzoni_nccp_nuova_compagnia_di_canto_popolare_7965/

testo_canzone_cicerenella_290287.html

6. Voir https://www.portanapoli.com/Ita/Teatro/cicerenella.html

7. Cf. De Filippo 1985, pp. 104-105.

8. Pour le texte, voir http://testicanzoni.mtv.it/testi-Pino-Daniele_8912/testo-\%27Na-tazzulella\%27e-caf\%C3\%A9-37160031

9. Voir https://www.rockol.it/testi/44769848/domenico-modugno-o-cafe

10. Voir https://www.monde-diplomatique.fr/mav/130/LEMOINE/51560

11. Voir https://ricerca.repubblica.it/repubblica/archivio/repubblica/1985/11/08/uccisiambrosoli-su-ordine-di-sindona-cosi.html)

12. Luzzato-Fegiz 1997.

13. Fasoli 2001, p. 68.

14. Giammusso 1993, p. 284.

15. https://www.rockit.it/articolo/neomelodici-tutto-quello-che-ce-sapere

16. https://www.controcampus.it/2014/02/neomelodici-significato-e-storia-il-fenomenoneomelodici-e-la-camorra/

17. ibidem

18. Voir http://testicanzoni.mtv.it/testi-Gianni-Vezzosi_13870924/testo-\%270-killer-10441512

19. Voir https://corrieredelmezzogiorno.corriere.it/napoli/notizie/cronaca/2011/17gennaio-2011/amico-camorrista-neomelodica-se-conosci-boss-non-giudichi-181274657827.shtml 20. https://www.peacelink.it/sociale/a/31498.html 


\section{ABSTRACTS}

Don Raffaè (1990) de De André/Bubola/Pagani, désormais un grand classique de la chanson napolitaine, décrit la société italienne des années 1990 à travers le regard du geôlier Pasquale Cafiero et de sa relation privilégiée, en prison, avec un boss mafieux, homme providentiel et magnanime, seul capable d'arranger les choses et d'aider les petites gens dans une société gangrenée par la corruption et l'individualisme.

Don Raffaè (1990) di De André/Bubola/Pagani, ormai un classico della canzone napoletana, descrive la società italiana degli anni 1990 attraverso lo sguardo del secondino Pasquale Cafiero e della relazione privilegiata che costui ha in prigione con un boss mafioso, uomo magnanimo e provvidenziale, unico in grado di sistemare le cose e d'aiutare il popolino in una società corrotta e malata d'individualismo.

\section{INDEX}

Chronological index: XXe siècle

Mots-clés: chanson napolitaine, Don Raffaè, De André, café, Ciccirinella, mafia, camorra Geographical index: Italie

\section{AUTHOR}

\section{WALTER ZIDARIČ}

Walter Zidarič est Professeur des universités en Littérature et Civilisation italiennes à l'Université de Nantes. IL est l'auteur de plusieurs ouvrages, en italien et en français, parmi lesquels on peut citer : L'univers dramatique d'Amilcare Ponchielli (Paris 2010), Fonti e influenze italiane per libretti d'opera del '900 e oltre (Lucca 2013), Tutto il teatro di Ercole Luigi Morselli (Roma 2017). Il codirige la collection Studi d'italianistica nel mondo éditée par les Presses universitaires de Louvain (PUL). Il est aussi librettiste : son Lars Cleen: lo straniero, tiré de la nouvelle Lontano de Luigi Pirandello, pour la musique de Paolo Rosato, a été représenté au Théâtre Metropolia de Helsinki en 2015. Son Orione (2018) pour la musique de Simone Fermani, tiré du drame homonyme de Ercole Luigi Morselli, sera mis en scène courant 2020. Il est l'auteur d'une pièce (ebook) Io, da qui, non me ne vado (2019), inspirée de la vie et des œuvres de Ercole Luigi Morselli. 